\title{
Editorial
}

\section{Artificial Intelligence and Dialysis}

\author{
Miguel Hueso $^{\mathrm{a}} \quad$ Alfredo Vellido $^{\mathrm{b}}$ \\ ${ }^{a}$ Department of Nephrology, Hospital Universitari Bellvitge and Bellvitge Research Institute (IDIBELL), L'Hospitalet \\ de Llobregat, Spain; ' Intelligent Data Science and Artificial Intelligence (IDEAI) Research Center, Universitat \\ Politècnica de Catalunya (UPC BarcelonaTech), Barcelona, Spain
}

Contemporary medical science heavily relies on the use of technology. Part of this technology strives to improve examination and measurement of the human body, with some of the most impressive technical breakthroughs to be found in the development of non-invasive procedures. Another part focuses on the development of devices that support therapies for specific pathologies. An example of this is the artificial kidney, which has become the target of intensive research from many directions and generates great expectations for dialysis patients. Research on the artificial kidney is still incipient though, and there are many challenges that must be overcome before it will become a reality and part of clinical practice in nephrology. One of these non-trivial challenges concerns the safety of users of these new dialysis devices. Safety risks make effective monitoring systems mandatory.

A byproduct of the abundance of technological devices surrounding the patient at the point of care is the continuous generation of data in electronic format, which, in turn, generates the need for analytical methods capable of transforming such data into knowledge that is ultimately useful for patients' treatment. The field of artificial intelligence (AI) is currently providing some of the most promising results as analytical tools.

\section{KARGER}

๑) 2018 S. Karger AG, Basel

E-Mail karger@karger.com

www.karger.com/kdd
AI is an umbrella term that conveniently shelters concepts from other fields such as statistics, algorithmics, machine learning (ML), information retrieval, and data science at large. It is precisely the advances in computational technologies that have enabled all fields of science to become data-rich environments. Medicine in particular is benefiting enormously from the advances resulting from data analysis using AI and related methods. The normalization of the use of electronic health records at the point of care allows these benefits to also permeate the healthcare sector.

The six papers in this issue of Kidney Diseases are the outcome of the Second Science for Dialysis Meeting: Artificial Intelligence, organized by the Hospital Universitari de Bellvitge in Barcelona, Spain, in which the medical and AI research communities gathered to discuss how applications of AI may improve the safety of artificial kidneys.

The current issue is organized in four parts. Part I is introductory and includes two papers: the first, by Hueso et al. [1], discusses the progresses and challenges of AI, ML, and medical decision support systems in their application to nephrology and to artificial kidneys; the second, by Vellido [2], delves into the many societal is- 
sues that face the application of AI in medical practice. Part II presents the concept of Reinforcement Learning, an area of ML inspired by goal-oriented algorithms which learn from interaction with the environment [3]. Part III includes two papers concerning AI and ML strategies to deal with blood pressure assessment and management by Ribas Ripoll and Vellido [4] and Barb- ieri et al. [5]. The former uses Deep Learning techniques while the latter involves end-stage kidney disease patients. Finally, part IV presents experience with artificial pancreas, currently introduced in clinical practice, by Mishina [6], involving the individualization in the response to dialysis treatment to improve dialysis dose accuracy.

\section{References}

1 Hueso M, Navarro E, Sandoval D, Cruzado JM. Progress in the development and challenges for the use of artificial kidneys and wearable dialysis devices. Kidney Dis. 2019; 5:3-10.

2 Vellido A. Societal issues concerning the application of artificial intelligence in medicine. Kidney Dis. 2019;5:11-17.
3 Jonsson A. Deep reinforcement learning in medicine. Kidney Dis. 2019;5:18-22.

4 Ribas Ripoll V, Vellido A. Blood pressure assessment with differential pulse transit time and deep learning: a proof of concept. Kidney Dis. 2019;5:23-27.
5 Barbieri C, Cattinelli I, Neri L, Mari F, Ramos $\mathrm{R}$, Brancaccio D, et al. Development of an artificial intelligence model to guide the management of blood pressure, fluid volume and dialysis dose in end-stage kidney disease patients: proof of concept and first clinical assessment. Kidney Dis. 2019;5:28-33.

6 Mishina S. Self-correcting abilities to improve accuracy of medical devices based on biofeedbacks. Kidney Dis. Forthcoming 2018. 